\title{
Monitoring quantum effects in cavity electrodynamics by atoms in semiclassical dressed states
}

\author{
G. S. Agarwal \\ School of Physics, University of Hyderabad, Hyderabad-500134, India
}

R. R. Puri

Theoretical Physics Division, Bhabha Atomic Research Centre, Bombay-400085, India

Received August 3, 1987; accepted April 5, 1988

\begin{abstract}
Field quantization effects in cavity electrodynamics can be isolated by sending through the cavity those atoms prepared initially in semiclassical dressed states. Under such initial conditions the fully quantized theory predicts irregular evolution, whereas the semiclassical and neoclassical theories predict, respectively, no dynamical evolution and regular periodic evolution.
\end{abstract}

Considerable attention has recently been focused on the quantum effects in the radiation-matter interaction. Prominent among these effects have been antibunching ${ }^{1}$ and the squeezing ${ }^{2}$ properties of the radiation field. In cavity electrodynamics ${ }^{3}$ one finds important manifestations of the quantum effects. ${ }^{4-6}$ These arise when an atom interacts in a high- $Q$ cavity with the field in a coherent state. One finds remarkable collapses and revivals ${ }^{4}$ of the Rabi oscillations. Note that if the field is treated classically, then the atomic excitation exhibits periodic oscillation as a function of time and there is no longer any collapse and revival of Rabi oscillations. Thus one has a mixture of semiclassical and quantum behavior, and, in general, it is difficult to isolate the part in the dynamics that is due to the quantized nature of the fields. Clearly, if there were a situation in which the atoms did not evolve if the field were treated as a given prescribed field, then any dynamical evolution would be a signature of the field quantization. In this paper we propose a method by which one can isolate the field quantization effects in the context of the cavity electrodynamics.

We would like to add that a quantized theory automatically includes the radiation-reaction effects. One could imagine including the radiation-reaction effects in a semiclassical way, as is done in the neoclassical theory. Use of neoclassical theory would lead to some dynamical evolution of the observables, even if these did not evolve if the fields were prescribed. Note that the neoclassical theory can be thought to be a decorrelated quantum theory, i.e., it includes the radiation-reaction effects but ignores the intrinsic quantum fluctuations. Thus our subsequent statements on the quantum nature refer to the inclusion of both radiation reaction and intrinsic quantum fluctuations. We will also show, in some cases, the differences in the dynamical evolution if the intrinsic quantum fluctuations are ignored.

Our proposal consists of sending an atom (assumed to be a two-level atom for simplicity) prepared in one of the two semiclassical dressed states through a resonant cavity. The semiclassical dressed states are defined to be the eigenstates of the Hamiltonian $H_{c}$ of an atom interacting with a semi- classical field. Thus the initial state $|\Psi(t=0)\rangle$ of the atom before it enters the cavity satisfies

$$
H_{c}|\Psi(t=0)\rangle=\lambda|\Psi(t=0)\rangle .
$$

If the field in the cavity is treated classically, then the interaction of the atom with the field in the cavity is given by $H_{c}$, and because the initial state is an eigenstate of $H_{c}$, it follows that the observables do not evolve:

$$
\langle\Psi(t)|G| \Psi(t)\rangle=\langle\Psi(0)|G| \Psi(0)\rangle,
$$

where $G$ is the operator corresponding to some physical observable, say, population inversion. On the other hand, the quantum nature of the field implies that $\langle G\rangle$ would evolve dynamically, and therefore the dynamics of $\langle G\rangle$ would be a signature of the quantum-mechanical nature of the field. This assumes that the transit time through the cavity is much less than the relaxation time. Note that the atom can be prepared in a semiclassical dressed state by suitably irradiating it with an external coherent field and by appropriately switching the phase ${ }^{7}$ of the field.

It should be noted that the present proposal is distinct from the usual studies in which the quantum effects of the radiation field are detected ${ }^{8}$ by the study of photon correlations or photon statistics. This is, for example, the case in the studies involving the antibunching and squeezing characteristics of the field that are seen in terms of the intensityintensity correlation functions of either the field or the field obtained by homodyning. Thus the present proposal is in a different class of measurements altogether.

We base our calculations on the Jaynes-Cummings model, ${ }^{9}$ which describes the interaction of a two-level atom with a single mode of the radiation field. The Hamiltonian in the interaction picture can be written as

$$
H_{q}=\hbar g\left(S^{+} a+S^{-} a^{+}\right) .
$$

We consider only the resonant situation and, for simplicity, assume a real coupling constant. The dynamics can be studied in terms of the eigenfunctions and eigenvalues of $H_{q}$, 
which are exactly known. ${ }^{10}$ The initial state of the field is assumed to be a coherent state $|z\rangle$, i.e.,

$$
\rho_{F}(0)=|z\rangle\left\langle z\left|=\sum_{m, n=0}^{\infty} \frac{z^{n} z^{* m}}{(n !)^{1 / 2}(m !)^{1 / 2}} \exp \left(-|z|^{2}\right)\right| n\right\rangle\langle m| .
$$

Only for such a state is a comparison of the results obtained with $H_{q}$ and the semiclassical Hamiltonian meaningful. The initial state of the atom is taken to be a coherent superposition of the ground and the excited states:

$$
\rho_{A}(0)=|\mu\rangle\langle\mu|; \quad| \mu\rangle=\left(1+|\mu|^{2}\right)^{-1 / 2}(|1 / 2\rangle+\mu|-1 / 2\rangle) \text {. }
$$

The semiclassical Hamiltonian $H_{c}$ corresponding to Eq. (3) is obtained by replacing the annihilation operator $a$ by a complex number $z$, i.e.,

$$
H_{c}=\hbar g\left(S^{+} z+S^{-} z^{*}\right) .
$$

The semiclassical dressed states corresponding to Eq. (6) are

$$
(1 / \sqrt{2})\left(\left|\frac{1}{2}\right\rangle \pm \frac{|g z|}{g z}\left|-\frac{1}{2}\right\rangle\right)
$$

These are thus the special cases of the superposition state [Eq. (5)] with $\mu$ equal to $\pm|g z| / g z$. Note that in the semiclassical dressed states the probability of finding the atom in the excited state is one half. Thus deviations in the excitation probability from a value of one half will be due to the quantum nature of the field. Using the eigenstates of Eq. (3) and the initial conditions (4) and (5), we have proved that the probability $P(t)$ of finding the atom in the excited state at time $t$ is given by

$$
\begin{aligned}
P(t)= & \frac{\exp \left(-|z|^{2}\right)}{2\left(1+|\mu|^{2}\right)} \sum_{n=0}^{\infty}\left(\frac { | z | ^ { 2 n } } { n ! } \left\{1-\frac{|z|^{2}|\mu|^{2}}{n+1} \cos \left[2 g t(n+1)^{1 / 2}\right]\right.\right. \\
& \left.+\frac{2|\mu||z|}{(n+1)^{1 / 2}} \sin (\theta+\varphi) \sin \left[2 g t(n+1)^{1 / 2}\right]\right\} \\
& \left.+\frac{|z|^{2 n}}{n !}\left[1+\frac{|\mu|^{2}|z|^{2}}{(n+1)}\right]\right) \\
\mu & =|\mu| \exp (i \varphi), \quad z=|z| \exp (i \theta) .
\end{aligned}
$$

Similarly, the complex dipole moment $\left\langle S^{-}\right\rangle$is found to be given by

$$
\begin{aligned}
D(t)= & \frac{\exp (i \omega t)}{4\left(1+|\mu|^{2}\right)} \sum_{n=1}^{\infty}\left(\exp \left\{i g t\left[(n+1)^{1 / 2}-(n)^{1 / 2}\right]\right\}\left(\mu \alpha_{n}+\alpha_{n-1}\right)\right. \\
& \times\left(\alpha_{n}{ }^{*}+\mu^{*} \alpha_{n+1}{ }^{*}\right)+\exp \left\{-i g t\left[(n+1)^{1 / 2}+(n)^{1 / 2}\right]\right\} \\
& \times\left(\alpha_{n-1}+\mu \alpha_{n}\right)\left(\alpha_{n}{ }^{*}-\mu^{*} \alpha_{n+1}{ }^{*}\right) \\
& - \text { terms with } g \rightarrow-g, \mu \rightarrow-\mu)+\frac{|\mu| \exp (i \omega t) \exp \left(-|z|^{2}\right)}{\left(1+|\mu|^{2}\right)} \\
& \times[\cos (g t) \exp (i \varphi)+i|\mu||z| \sin (g t) \exp (-i \theta)] \\
& \alpha_{n}=\frac{z^{n} \exp \left(-|z|^{2} / 2\right)}{(n !)^{1 / 2}} .
\end{aligned}
$$

On the other hand, if the field in the cavity is treated semiclassically $(a \rightarrow z)$, i.e., if the interaction in the cavity is described by the Hamiltonian of Eq. (6), then the corre- sponding results for the excitation probability and the dipole moment are

$$
\begin{aligned}
P(t)= & 1 / 2+\left[\left(1-|\mu|^{2}\right) \cos (2 g|z| t)+2|\mu| \sin (\theta+\varphi)\right. \\
& \times \sin (2 g|z| t)] /\left(1+|\mu|^{2}\right), \\
& \stackrel{\text { for } \theta+\varphi=0, \pi,}{\longrightarrow} \begin{cases}1 / 2 & \\
1 / 2[1+\sin (2 g t|z|)] & \text { for } \theta+\varphi=\pi / 2\end{cases}
\end{aligned}
$$

$$
\begin{aligned}
D(t)= & \frac{\exp (-i \theta)}{\left(1+|\mu|^{2}\right)}[|\mu| \cos (\theta+\varphi)+i|\mu| \sin (\theta+\varphi) \cos (2 g t|z|) \\
& \left.-\frac{i\left(1-|\mu|^{2}\right)}{2} \sin (2 g|z| t)\right]
\end{aligned}
$$

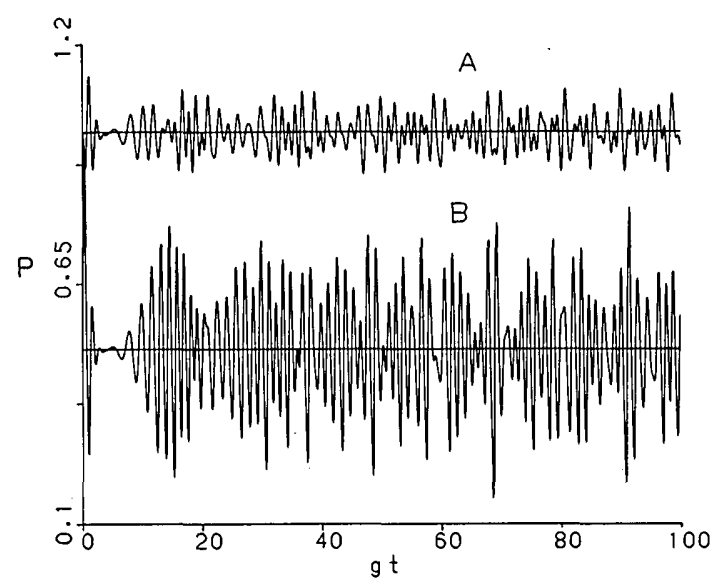

(a)

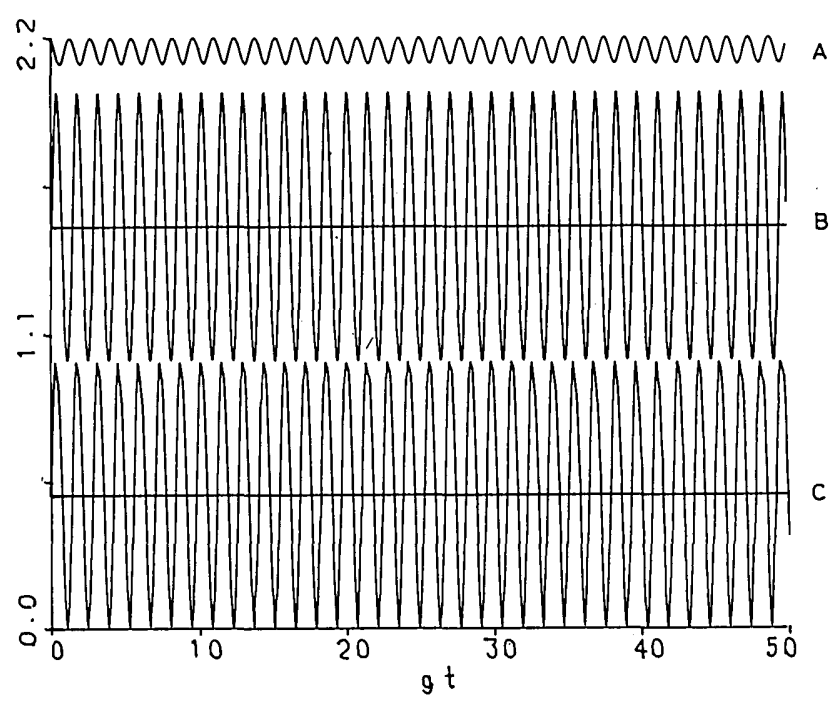

(b)

Fig. 1. The probability $P(t)$ of finding the atom in the excited state as a function of $g t$ for an atom initially prepared in a coherent superposition [Eq. (5)], with $|\mu|=1$. Curves $A$ and B in (a) give, respectively, $2 P(t)$ and $P(t)$ for $\varphi=0$ and $\varphi=\pi / 2$, respectively. (b) The corresponding predictions of the semiclassical (trace $\mathrm{C}$, for $\varphi=$ $\pi / 2)$ and the neoclassical (trace $\mathrm{A}[2 P(t)+2.2]$ and trace $\mathrm{B}[P(t)+1]$ for $\varphi=0$ and $\varphi=\pi / 2$, respectively\} theories. The average number $|z|^{2}$ of cavity photons is 5 , and $\theta=0$. 


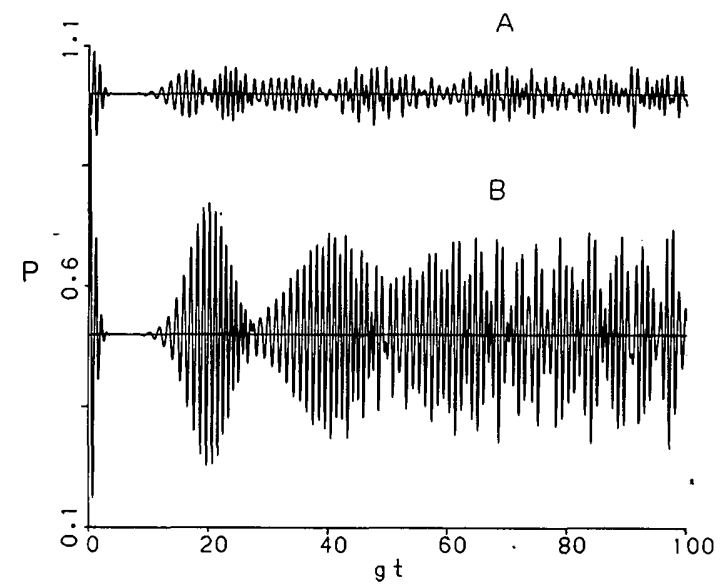

(a)

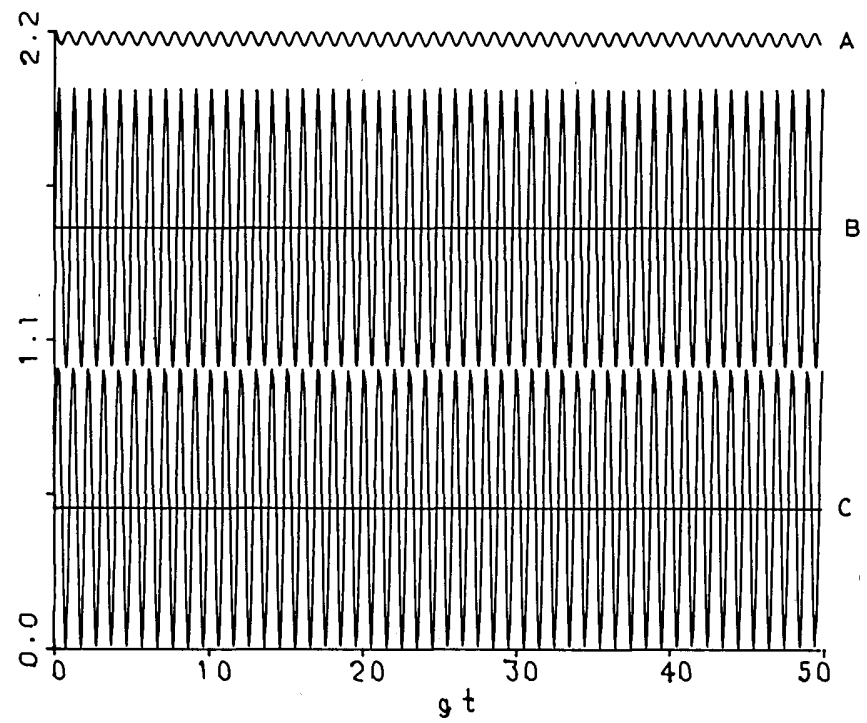

(b)

Fig. 2. The same as Fig. 1 but with an average number of cavity photons of 10 .

$$
\stackrel{|\mu|=1}{\longrightarrow}\left\{\begin{array}{ll}
1 / 2 & \text { for } \theta+\varphi=0 \\
(i / 2) \cos (2 g|z| t) & \text { for } \theta+\varphi=\pi / 2
\end{array} .\right.
$$

For large $|z|^{2}$ asymptotic expansion of the excitation probability can be carried out as usual. One finds, for example, the following result at the $k$ th revival time $(g t / 2 \pi|z|=k)$ :

$$
\begin{aligned}
P(k)= & \frac{1}{2}\left[1+\frac{\left(1-|\mu|^{2}\right) \cos \left(2 \pi|z|^{2} k+1 / 2 \tan ^{-1} \pi k\right)}{\left(1+\pi^{2} k^{2}\right)^{1 / 4}\left(1+|\mu|^{2}\right)}\right] \\
& \rightarrow 1 / 2 \quad \text { for }|\mu|=1 .
\end{aligned}
$$

In Figs. 1 and 2 we show the dramatic changes in the dynamics of the atom as we change the parameter $\varphi$ for $\theta=0$, $|\mu|=1$. For $\varphi \neq 0$ or $\varphi \neq \pi$, the initial coherent state of the atom evolves even if the cavity field is treated classically. Thus, for $\varphi \neq 0$ or $\varphi \neq \pi$, we have evolution due to both the classical nature and the quantum nature of the field.

However, for $\varphi=0$ or $\varphi=\pi$ there is no dynamical evolution of the initial state if the field in the cavity is treated classically, for then the initial state is an eigenstate of Eq.
(6). Therefore the dynamical evolution as displayed by traces A in Figs. 1(a) and 2(a) is due purely to the quantum nature of the cavity field. Thus the effects due to the quantum nature of the field in the cavity can be isolated by setting the initial phase $\varphi$.

Note that in the quantum theory the reaction of the atoms on the field is automatically included through what we might call the operator reaction field. We might ask what happens if the effects of backreaction are included in a classical way. We can use the neoclassical theory to investigate the dynamical evolution of the atoms prepared in semiclassical dressed states. We have proved that the neoclassical equation for the inversion $X=\left\langle S^{z}\right\rangle$ is

$$
(\dot{X})^{2}-4 g^{2} X^{3}+4 g^{2}|z|^{2} X^{2}+g^{2} X=|z|^{2} g^{2} \sin ^{2}(\theta+\varphi) \text {. }
$$

This equation is easily integrated numerically for different values of the phase settings. In Figs. 1(b) and 2(b) we show

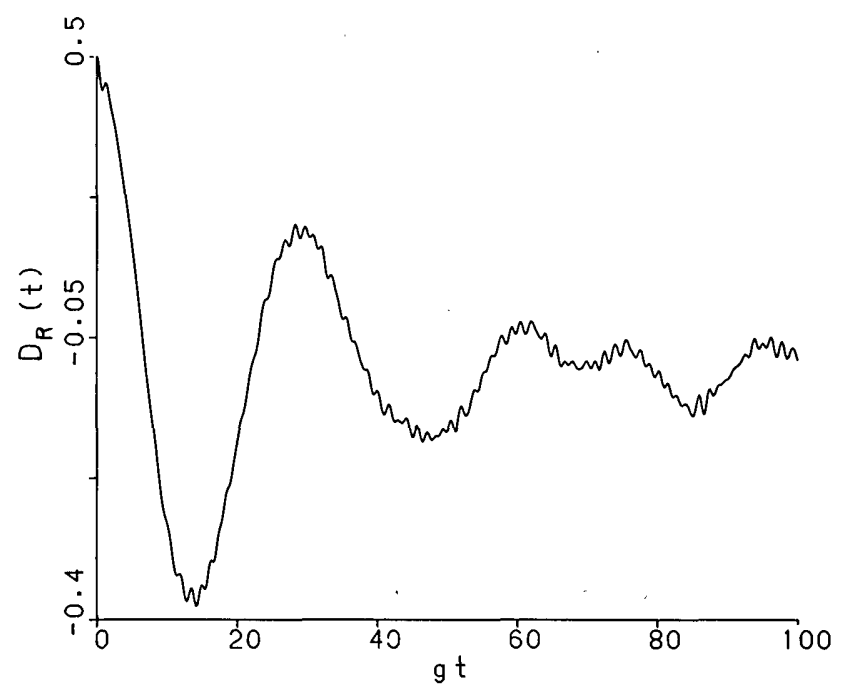

Fig. 3. The real part $D_{R}(t)$ of the complex dipole moment as a function of $g t$ for $|\mu|=1,|z|^{2}=5$, and $\theta=\varphi=0$.

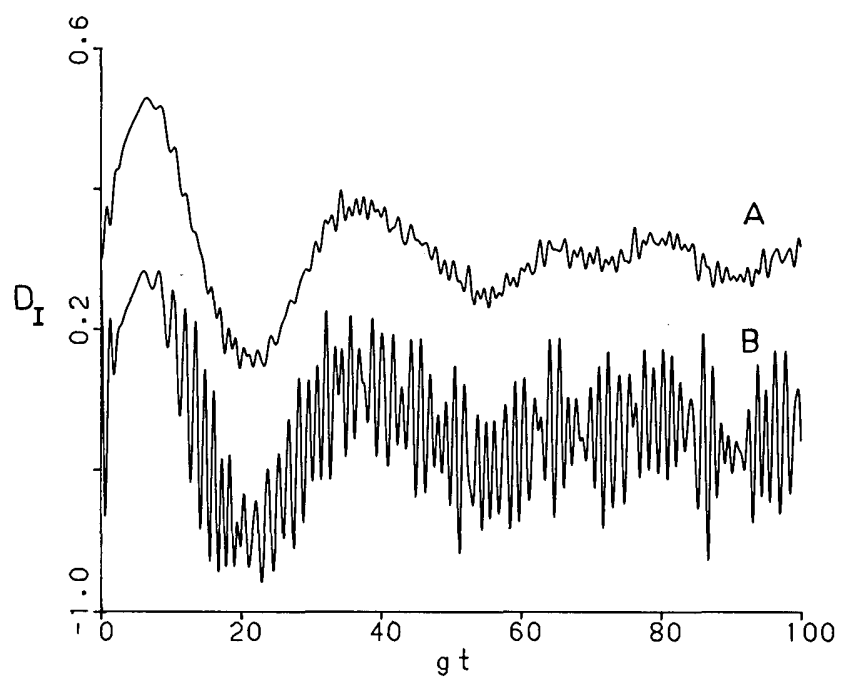

Fig. 4. The imaginary part $D_{I}(t)$ of the dipole moment as a function of $g t$ for $|\mu|=1,|z|^{2}=5, \theta=0$ : trace $\mathrm{A}, \varphi=0$; trace $\mathrm{B}, \varphi=\pi / 2$. The origin on the $y$ axis for trace $B$ has been shifted by $-1 / 2$ unit. 


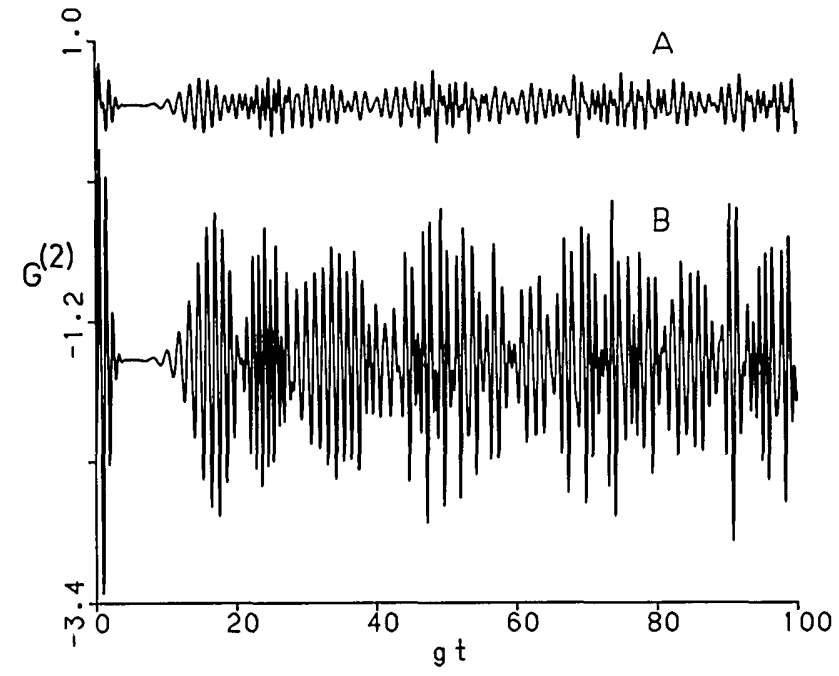

Fig. 5. The photon-number fluctuation parameter $G^{(2)}(t)=$ $\left\langle a^{+2}(t) a^{2}(t)\right\rangle-\left\langle a^{+}(t) a(t)\right\rangle^{2}$ as a function of $g t$ for $|z|^{2}=10,|\mu|=1, \theta$ $=0$ : $\operatorname{trace} \mathrm{A}, \varphi=0 ;$ trace $\mathrm{B}, \varphi=\pi / 2$. The origin on the $y$ axis for the curve $B$ has been shifted by -2 units.

the dynamical evolution as predicted by the neoclassical and semiclassical theories. For large $|z|^{2}$ the neoclassical results come close to the semiclassical results. It can be proved that

$$
\begin{aligned}
& P(t)=\frac{1}{2}-\frac{1}{4|z|^{2}} \sin ^{2}(g t|z|) \quad\left(\text { for } \theta=\varphi=0, \quad|z|^{2} \gg 1\right) \\
& =1 / 2[1+\sin (2 g t|z|)] \quad\left(\text { for } \theta=0, \quad \varphi=\frac{\pi}{2}, \quad|z|^{2} \gg 1\right) .
\end{aligned}
$$

This is also borne out by Figs. 1(b) and 2(b). Thus, for $\theta+\varphi$ $=0$, the regular motion predicted by these theories is to be contrasted with the irregular motion predicted by the theory in which the field is quantized. It should be kept in mind that the above conclusions rely on the assumption that all sources of incoherence are insignificant, i.e., that the cavity has a high- $Q$ value and that the transit time is much smaller than the relaxation time.

In Figs. 3 and 4 we display the time evolution of the real and imaginary parts of the dipole moment for various phase settings. The dynamical changes in the dipole moment for $\theta$ $=\varphi=0$ and $|\mu|=1$ are due to the quantum nature of the field in the cavity. Note that the real part of the dipole moment is zero for $\varphi=\pi / 2$. Thus the behavior shown in Fig. 3 is an effect that is due to the quantization of the radiation field. A comparison of Figs. 1 and 2 also shows that the quantum effects become less pronounced with an increase in the average photon numbers in the cavity. The phase of $\mu$ is fixed by the phase $\theta_{0}$ of the coherent microwave field (used for the initial preparation of the atom) $\varphi=\pi / 2-\theta_{0}$. We can imagine that the cavity is driven by the same microwave field but is much attenuated and phase shifted by $\pi / 2$ so that $\theta=\theta_{0}-\pi / 2$.
The properties of the cavity field are also quite sensitive to the initial preparation of the atomic system. For example, the photon-number fluctuation parameter $G^{(2)}(t)$, defined as

$$
G^{(2)}(t)=\left\langle\left[a^{+2} a^{2}(t)\right]\right\rangle-\left\langle a^{+}(t) a(t)\right\rangle^{2},
$$

can be evaluated. We do not present an explicit expression for $G^{(2)}$, as it is rather long. Figure 5 shows the dependence of $G^{(2)}(t)$ on the initial atomic coherence. The quantum fluctuations in the cavity field are more pronounced in the case when the atom evolves, even in the presence of a semiclassical field. This is connected with the operator conversation law $S^{z}+a^{+} a=$ constant. This conversation law implies that the quantum fluctuations in the atomic and field variables are complimentary to each other. Note that so far it has been feasible to study experimentally, in connection with Rydberg atoms, the dynamics of the atom rather than the dynamics of the cavity field.

In conclusion, we have presented a method based on the study of the atomic dynamics that will allow us to probe the effects of the quantized nature of the field in the cavity.

\section{ACKNOWLEDGMENTS}

G. S. Agarwal is grateful to J. H. Eberly, M. O. Scully, and H. Walther for discussions on the subject matter of this paper.

\section{REFERENCES AND NOTES}

1. See, for example, reviews by R. Loudon, Rep. Prog. Phys. 48, 58 (1980); H. Paul, Rev. Mod. Phys. 54, 1061 (1982); D. F. Walls, Nature 280, 451 (1979).

2. D. F. Walls, Nature 306, 141 (1983); C. M. Caves and B. L. Schumaker, Phys. Rev. A 31, 3068, 3093 (1985), and references therein.

3. S. Haroche and J. M. Raimond, in Advances in Atomic and Molecular Physics, D. R. Bates and B. Bederson, eds. (Academic, New York, 1985), Vol. 20, p. 3347.

4. J. H. Eberly, N. B. Narzohny, and J. J. Sanchez-Mondragon, Phys. Rev. Lett. 44, 1323 (1980); H. I. Yoo and J. H. Eberly, Phys. Rep. 118, 239 (1985)

5. S. Stenholm, Phys. Rep. C 6, 1 (1973); P. Meystre, E. Geneux, A. Quattrapani, and A. Faist, Nuovo Cimento B 25, 21 (1975); S. Singh, Phys. Rev. A 25, 3206 (1982).

6. G. S. Agarwal, Phys. Rev. Lett. 53, 1732 (1984); J. J. SanchezMondragon, N. B. Narozhny, and J. H. Eberly, Phys. Rev. Lett. 51,550 (1983).

7. Y.S. Bai, A. G. Yodh, and T. W. Mossberg, Phys. Rev. Lett. 55, 1277 (1985); J. Krause, M. O. Scully, and H. Walther [Phys. Rev. A 34, 2032 (1986)] have considered coherent preparation of atoms and have examined Ramsay-like situations.

8. H. J. Kimble, M. Dagenais, and L. Mandel, Phys. Rev. Lett. 39 , 691 (1977); L. Mandel, Phys. Rev. Lett. 49, 136 (1982); R. Short and L. Mandel, Phys. Rev. Lett. 51, 384 (1983).

9. E. T. Jaynes and F. W. Cummings, Proc. IEEE 51, 89 (1963).

10. Present experiments are in a regime [G. Rempe, $H$. Walther, and N. Klein, Phys. Rev. Lett. 58, 353 (1987)] in which the effect of cavity damping is not important. However, if necessary, the effects of cavity damping can be accounted for by using the method of R. R. Puri and G. S. Agarwal, Phys. Rev. A 33, 3610 (1986); see also S. M. Barnett and P. L. Knight, Phys. Rev. A 33, 2444 (1986). 